\title{
РАЗДЕЛ IV. МАТЕМАТИКА
}

\section{Ванчиков В.Ц., Грушко В.М., Данеев Р.А. \\ Константа $2 \pi$ в математических моделях процессов различной природы}

Восточно-Сибирский институт МВД России (Россия, Иркутск)

doi: $10.18411 / s r-10-12-2018-21$

idsp: sciencerussia-10-12-2018-21

При изучении различных процессов в работе сделана попытка связать воедино некоторые явления и объяснить глубинные вопросы о природе физической реальности на основе единого фундамента мироздания - электрического заряда.

Так, в гидродинамике при исследовании капилляров при числе Рейнольдса,

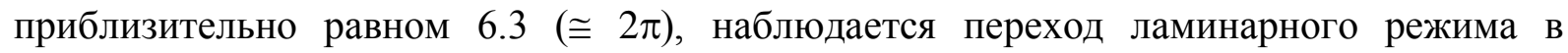
разновидность слоистого течения, сопровождаемое прилипанием молекул потока к стенкам капилляров. При этом жидкость организуется в виде многослойной неподвижной пленки граничного слоя на твердой поверхности. Обратная величина числа Рейнольдса, отражающая поведение движущейся жидкости в терминах относительной роли вязкого трения и инерционного отклика $(1 / 6,3 \approx 1 / 2 \pi)$, приблизительно равная 0.16, при которой происходит вышеуказанный переход, подкрепляется аналогией с уровнем протекания в трехмерных задачах теории перколяции [1]. При движении жидкости в трубах тормозящее влияние силы адгезии стенок распространяется на все большую толщу потока. На некотором участке от входа устанавливается параболическое распределение скорости. Для длины начального участка в плоской трубе Г. Шлихтинг получил

$$
\ell_{\text {нач }}=0,16 \text { a } \mathrm{Re},
$$

где $a$ - расстояние от оси потока до стенки трубы.

Для длины начального участка в круглой трубе О. М. Тарг получил зависимость

$$
\ell_{\text {нач }}=0,16 \mathrm{r}_{\mathrm{o}} \mathrm{Re} \text {, }
$$

где $\mathrm{r}_{\mathrm{o}}$ - радиус трубы. Этот результат теоретического расчета обнаруживает хорошее соответствие с данными обстоятельных опытов И. Никурадзе [2].

Продолжая аналогию с проявлениями сил адгезии твердой поверхности, приведем результаты Л.Д. Ландау для крыла в виде плоской бесконечно большой пластины, где коэффициент подъемной силы $\mathrm{C}_{\text {y }}$ равен произведению угла атаки $\alpha$ на постоянную составляющую, равной $2 \pi[3]$.

$$
\mathrm{C}_{\mathrm{y}}=2 \pi \cdot \alpha \text {. }
$$

Расчеты, выполненные Н.Е. Жуковским в терминах вихревого движения реальной жидкости, подтверждают правомерность формулы (1). При этом ее экспериментальная проверка показала, что для достаточно тонких крыловых профилей, указанная формула приближенно применима при малых углах атаки [2].

Расчеты, выполненные Я.И. Френкелем в терминах проявления упругой силы в идеальном кристалле, показали, что отношение модуля сдвига $G$ к сдвиговой прочности $\mathrm{G}_{\mathrm{c}}$ равно

$$
\mathrm{G} / \mathrm{G}_{\mathrm{c}}=2 \pi .
$$

Иными словами, необходимо преодолевать упругое сопротивление, величина которого при деформации изменяется периодически, повторяясь на межузловое расстояние решетки кристалла. Этот вывод экспериментально подтверждается атомносиловым микроскопом, где период осцилляций силового взаимодействия кончика иглы кантилевера совпадает с периодом кристаллической решетки [4]. 
Множитель 0,16 наблюдается также в электродинамике, когда формула силы взаимодействия двух проводников тока включает в себя множитель $1 / 2 \pi$, не входящий в саму константу коэффициента пропорциональности. Конкретная причина появления этого иррационального множителя в работе [5] не уточняется и объясняется либо геометрическими свойствами пространства либо свойствами источников тока.

В статической ситуации О. Хевисайд предложил рационализировать записи расчетов по формуле Ш. Кулона, описывающую силу взаимодействия между двумя точечными электрическими зарядами, введением множителя $1 / 4 \pi$ (или 0,16/2). В результате сокращения множитель $4 \pi$ исчезает из наиболее употребительных формул, делая их простыми. Однако «невероятная эффективность математических расчетов в естественных науках, граничащая с мистикой» [6], приводит к появлению отмеченного множителя в других формулах, делая расчеты неудобными. Возможно, что существует пока еще не открытый «механизм» и что детали внутреннего сложного устройства скрыты в уравнениях. Это может быть как математический принцип, утверждающий, что существует некая иррациональная величина, приблизительно равная $2 \pi$, проявляющаяся в частности в физике критических явлений. И, несмотря на отличное согласие с теоретическими и экспериментальными результатами из теории перколяции, нет еще отчетливого понимания этого нечто.

Вещество (проводник тока, труба, капилляр, крыло и т.д.) состоит из частиц атомных размеров. Поведение атомных объектов (электрон, протон, нейтрон, фотон и т.д.) одинаково: всех их можно назвать «частицами-волнами». С этих позиции упругое сопротивление деформации сдвига неподвижной пленки граничного слоя жидкости, кристалла - это не классический, квантовомеханический эффект. Истинная причина упругой силы в том, что при сдвиговой деформации атомы сминаются и электроны (волны определенной длины) вынуждены тесниться в меньшем объеме. Поскольку электроны атома стараются держаться подальше друг от друга, то (по принципу неопределенности - неопределенность в $\mathrm{k}$ равна $2 \pi / \mathrm{L}$, где $\mathrm{k}$ - волновое число, $\mathrm{L}$ - длина цуга волн) их импульсы в среднем увеличиваются, а увеличение импульсов означает рост энергии. А по информационной модели состояние сминаемого атома передается в виде волн де Бройля. В результате происходит обмен информацией между электронами (то есть обмен информацией связан с обменом энергией).

Во многих случаях в математических моделях процессов, описывающих электромагнитные, перколяционные и другие явления, появляется иррациональный коэффициент, равный $2 \pi$ (или $1 / 2 \pi$ ). В то же время, для увеличения «эффективности математических расчетов» в наиболее употребительных формулах электродинамики это число сокращают (рационализацией законов взаимодействия электрических зарядов). Несмотря на эту процедуру, вышеуказанное число появляется в других формулах. При действии упругих и адгезионных сил постоянная электромагнитного взаимодействия определяет конфигурацию электронных оболочек и прочность химических, a также молекулярных связей. $\mathrm{B}$ результате чего возникает квантовомеханический эффект энергоинформационного обмена между электронами (то есть как следствие процессов, происходящих в спиновых микросистемах). Однако детали информационного содержания данного явления в дальнейшем требует экспериментального и теоретического уточнения.

$$
* * *
$$

1. Эфрос А.Л. Физика и геометрия беспорядка. М.: Наука, 1982. С.127. С.118.

2. Емцев Б.Т. Техническая гидромеханика. М.: Машиностроение, 1978. С.393. С.259.

3. Ландау Л.Д., Лифшиц Е.М. Теоретическая физика. Т.6. Гидромеханика. М.: Наука, 1988. С. 736.

4. Springer Handbook of Nanotehnogy (ed. By Bhushan). Berlin: Springer-Verlag. 2004. 1222 p.

5. Суорц К.Э. Необыкновенная физика обыкновенных явлений. Т.2. М.: Наука, 1987. С.221.

6. Wigner E.P. The Unreasonable Effectiveness of Mathematics in the Natural Sciences. Comm. Pure and Appl. Math. 131.1(1960). 